\section{International Journal of New Trends in Social Sciences}

Volume 3, Issue 1, (2019) 010-016

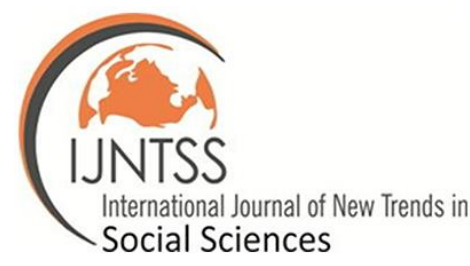

www.ij-ss.eu

\title{
Integrating Microsoft loT, machine learning in a large-scale power meter reading
}

Liviu Pascu*, Politehnica University of Timisoara, Vasile Parvan No. 2, 300223 Timisoara, Romania

Attila Simo, Politehnica University of Timisoara, Vasile Parvan No. 2, 300223 Timisoara, Romania

Alin Mihai Vernica, Politehnica University of Timisoara, Vasile Parvan No. 2, 300223 Timisoara, Romania

\section{Suggested Citation:}

Pascu, L., Simo, A. \& Vernica, A. M. (2019). Integrating Microsoft loT, machine learning in a large-scale power meter reading. International Journal of New Trends in Social Sciences. 3(1), 010-016. https://doi.org/10.18844/ijntss.v3i1.3815

Received from August 10, 2018; revised from January 05, 2019; accepted from May, 20, 2019.

Selection and peer review under responsibility of Prof. Dr. Huseyin Uzunboylu, Near East University, Cyprus.

${ }^{\circ} 2019$ United World Center of Research Innovation and Publication. All rights reserved.

\begin{abstract}
Due to fast technological progress in the power engineering field, the need of new information/communication technologies is more and more underlined. e-Learning has become a viable alternative to traditional teaching/learning techniques, adopted especially due to the advantages offered by the possibility of continuous training. This paper presents a Microsoft internet of things platform for a very large-scale smart power meter reading, used not only for training operative staff of the distribution network operator but also to help end users to control electrical energy that they consume. The strength of this platform for the distribution network operator is that the read data can be used for energy forecast, which is very useful for the future energy consumption optimisation. The platform can be reached through the Internet using a user name and password. A comparison between the results provided by classical teaching/learning methods and the ones achieved using this platform is presented.
\end{abstract}

Keywords: Machine learning, internet of things (IoT), training.

\footnotetext{
* AdDRESS FOR CORRESPONDENCE: Liviu Pascu, Politehnica University of Timisoara, Vasile Parvan No. 2, 300223 Timisoara, Romania. E-mail address: liviupascu@upt.ro / Tel.: +4-025-640-3430
} 


\section{Introduction}

In the past period, many improvements were done in the metering area. The new digital meters are capable of storing much more information concerning how the end user is consuming the energy and are capable to automatically or on request transfer this information to the energy distrusters. These new digital meters are called smart meters because they are offering the possibility to the energy distributer: to better understand the needs of the customers, to better invoice the exact consumption and to better forecast the future consumption.

In the same time, in order to be able to use the information possible to obtain from these smart meters, new platforms were developed to be able to connect to smart meters, to store the historical data and to manipulate the received information. One of these new platforms provide the capability to connect, store and manipulate the information received from smart meters in Microsoft internet of things (loT) platform.

This paper presents a training system that uses the Microsoft loT platform and a set of simulated hourly energy consumption data set (similar with the ones possible to obtain from smart meters) that predicts the energy consumption for the next day to train students or distribution network operators on how to use such a system. As a lot of effort and investment is put in the next years to utilise as many end users as possible with smart meters, as future utilisation of the actual system with minimal effort, the simulated data set can be replaced with the information received from real smart meters and the prediction offered by the system can help the distribution network operators to better manage and plan the actions needed to be done to operate their networks in an optimal way (JigoriaOprea, Simo \& Borlea, 2014).

\section{System description}

\subsection{Integration in real environment}

The actual situation and future plans to have a smart meter for each end user are using a very hybrid solution in terms of the types of equipment. There are smart meters that are accessible over IP, GSM or radio. The actual situation and planned one propose that concentrators are used to communicate with smart meters and provide the received information to the next systems that will store and manipulate the data sets. Most of the concentrators are compatible with industry standards, but still some of the equipment will have native integration with Microsoft loT platform and some will do not. For the real environment, our proposed solutions take into account this multitude of equipment that will exist in the field and propose a solution that will be able to handle the situation. In Figure 1, the main components of the proposed solution and how they interact are presented (Metering, 2015).

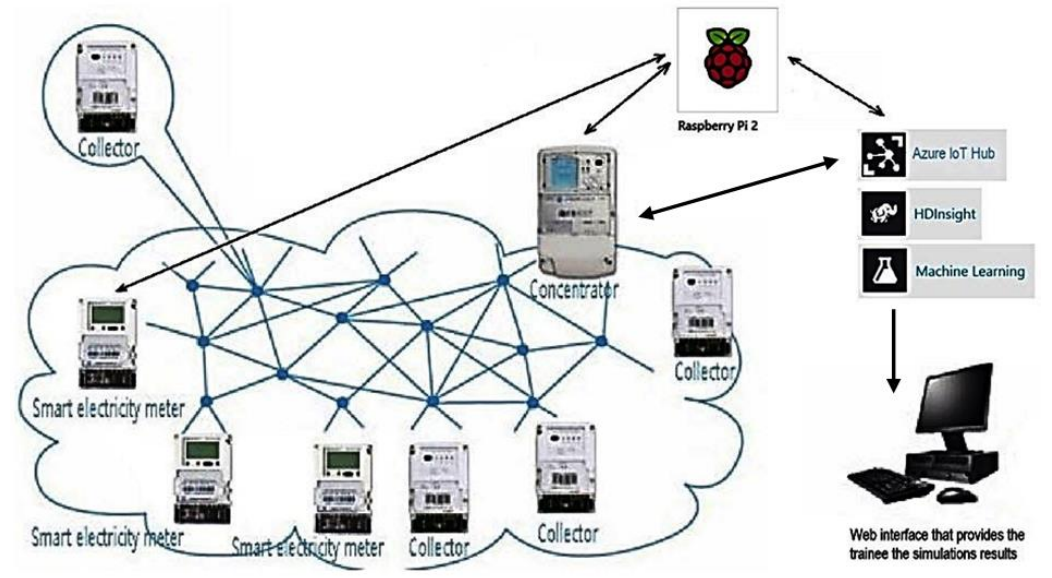

Figure 1. System main components and interaction in real environment 


\subsection{Integration in training environment}

In the training environment, the connectivity with the real smart meters and concentrators was replaced with a computer programme that simulates the measurements from 100,000 smart meters. In Figure 2, the main components of the training solution are presented.

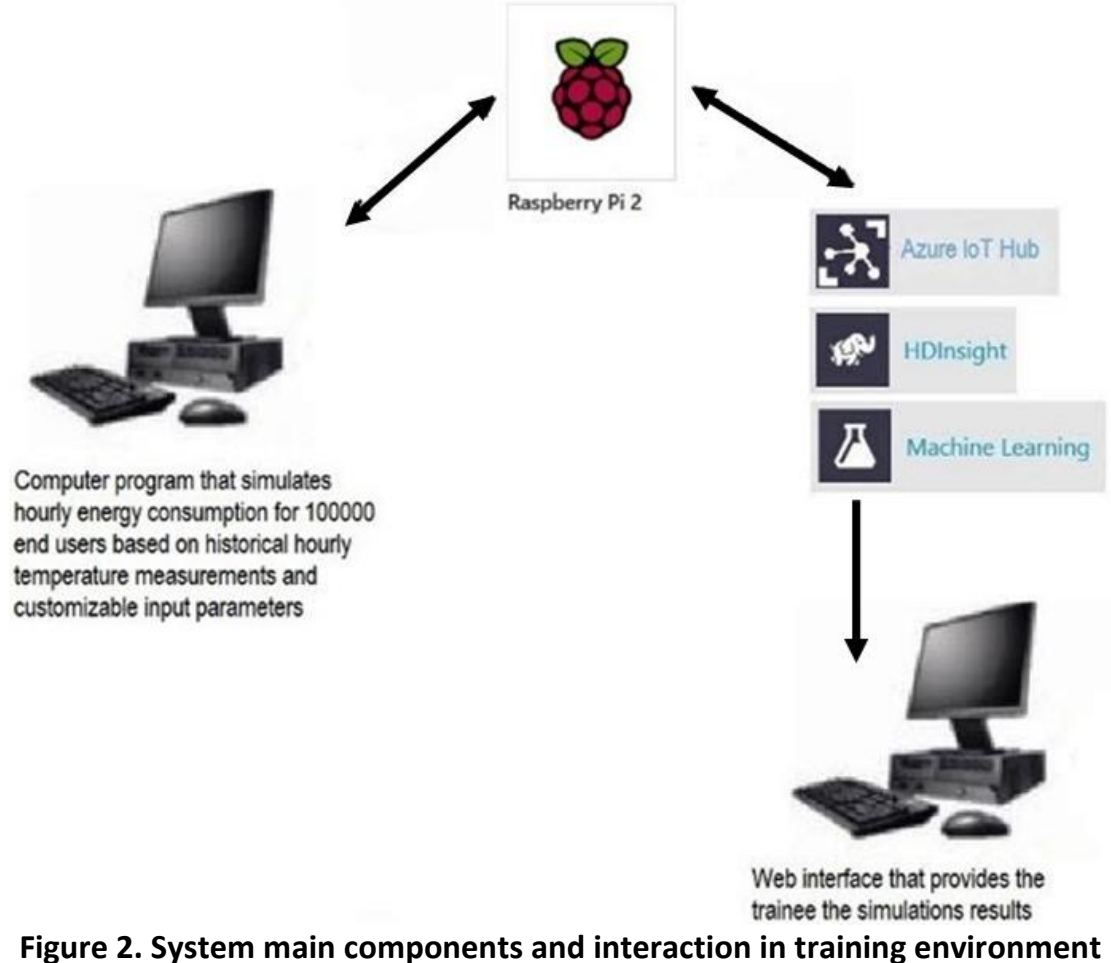

The programme that simulates the measurements from 100,000 smart meters is generating the hourly consumption based on the following changeable inputs and unchangeable inputs:

Changeable inputs:

- Types of equipment used and their consumption dependence on the outside temperature.

- Percentages of consumer types from the total number of estimated consumers. We use eight consumer types in our simulations.

- Unchangeable inputs:

- Historical hourly temperature measurements obtained from specialised websites for the past 365 days.

The simulated data set is consumed by the Raspberry Pi equipment and transferred to the Azure loT Hub, stored in a HDInsight Data Lake service and analysed by a predictive analytic solution implemented in a Machine Learning Azure service that was based on the data set received from Raspberry Pi device, and the temperature prognoses for the next day is providing an estimated hourly energy consumption. Finally, the results are presented to the trainee on a web interface (Historical Temperature Measurements, 2016).

Some examples of the parameter used by simulation programme are presented in Figure 3 (Next Day Prediction, 2016). 


\subsection{Advantages for using the training system}

The following advantages are to be mentioned:

- Trainees are able, in a simple way, to simulate different environments

- Trainees are able to understand what are the major factors that can influence the prognoses of consumption

- In a hybrid environment (part of the end users is covered by smart meters and part not), based on the information received from the existing smart meters, an extrapolation can be made to better predict the next day consumption and by doing this manage in an more optimised way of the distribution networks

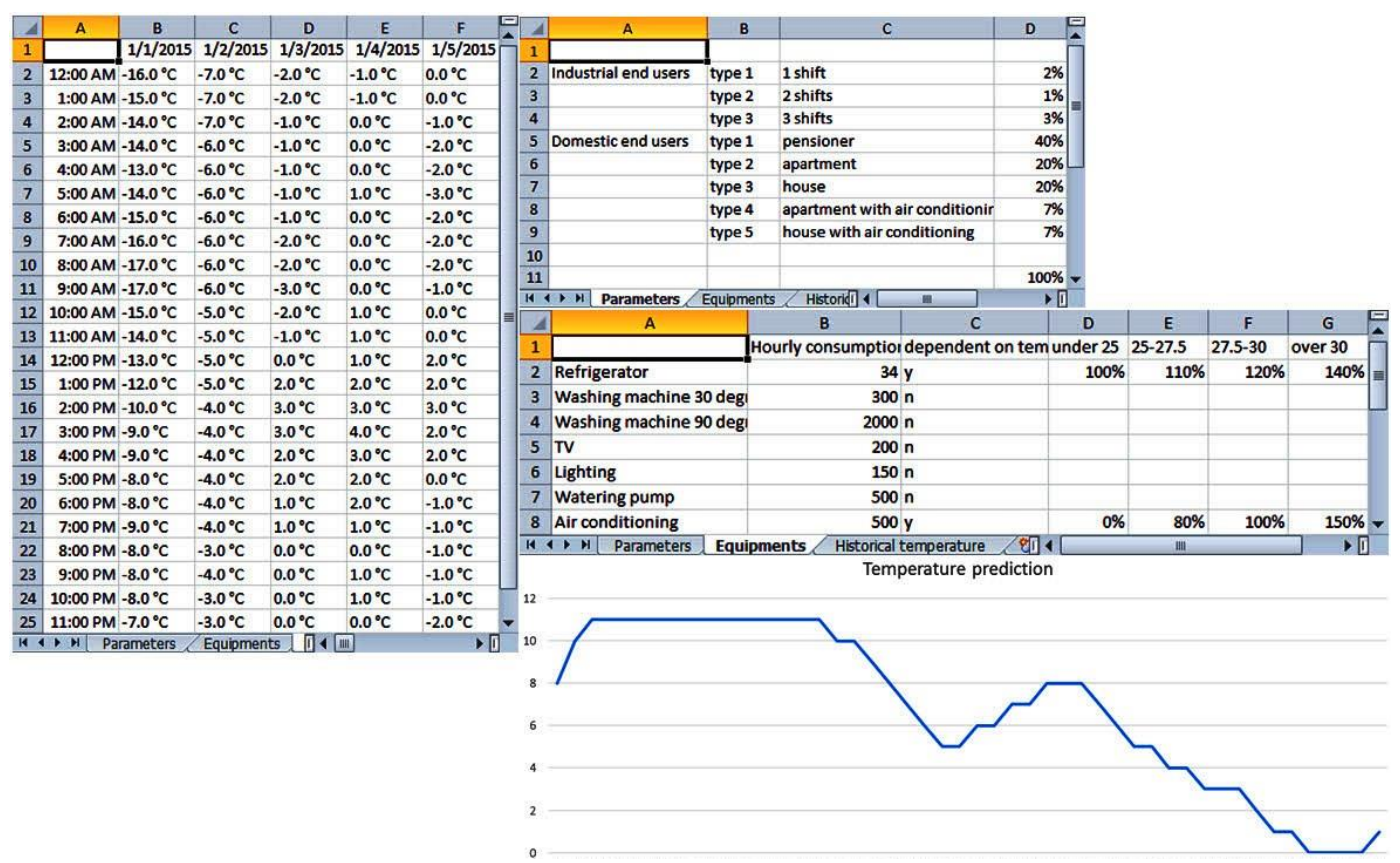

Figure 3. Parameters used by the simulation programme

\section{Case study}

The assumption on which our case study is based is that plausible results can be obtained by applying modern teaching/learning techniques for operative staff training, regarding the use of the developed platform and its features. Furthermore, this case study shows that results are better if modern and traditional methods are combined. Researcher, the aim of the study was to validate a prototype of a game-based educational tool for improving auscultation skills (Coskun, Adiguzel \& Catak, 2019).

The participants, in this study, are the operative staff of the distribution network operator. There were 60 participants: $24 \%$ of women and $76 \%$ of men. Their average age was 36 years old. The participants were divided into three groups of 20 people, heterogeneous in terms of their levels of knowledge.

The study lasted 6 weeks, during which 60 participants were divided into three groups of 20 (OS1, OS2 and OS3). Individuals from the group OS1 attended a training session, where the information content was submitted by the traditional mosaic method. The mosaic method is used for the practice of individual and collaborative learning. It is used by experts as an aid for teaching and requires a flexible algorithmic process within small activity groups (Rosenberg, 2011). The whole group OS1 was divided 
into small groups of four, each time in a different order to change their roles and tasks (Simo, Barbulescu \& Kilyeni, 2014).

The steps in this process are as follows:

a) The formation of heterogeneous groups;

b) The presentation of the topic and its division into sub-themes;

c) The formation of expert groups, presentation of instructions and clarification of tasks;

d) The study of material in the experts' groups and preparation of responses to those in the home or original group (this is the time for experts to teach their teammates);

e) The restoration of the original groups and the presentation of answers to the teams of experts;

f) Individual testing;

g) Assessment of teamwork and recognition of their merits;

h) Summarise the position and arguments of a work group and the writing of an individual work.

Group OS2 received the material electronically, tutorials and visual demonstrations. During this period of the study, they had never seen any trainer. The participants studied by themselves through the informational content and solved a number of the proposed problems. This kind of approach is also the case of end users, which after the platform launch on Internet will not have access to trainer, only to tutorials.

Group OS3 also received the material in an electronic format, the same content as participants in the group OS2. However, after an independent study, the forum platform was available for online questions addressed to trainers or other participants.

At the end of this training period, a final evaluation was done to measure the level reached by each group and each participant and compare the results obtained by different teaching-learning techniques. The grades of participants were divided into three categories: small grades representing the grades in the [1-4] range, medium grades representing the grades in the [5-7] range and high grades representing the grades in the [8-10] range.

\section{Results and discussions}

The obtained results are synthesised in Table 1.

Table 1. Results

\begin{tabular}{lccc}
\hline \multicolumn{1}{c}{ Grades } & Group OS1 & Group OS2 & Group OS3 \\
\hline High [8-10] & $44 \%$ & $28 \%$ & $47 \%$ \\
Medium [5-7] & $8 \%$ & $48 \%$ & $45 \%$ \\
Small [1-4] & $48 \%$ & $24 \%$ & $8 \%$ \\
\hline
\end{tabular}

For a better understanding, the grade distribution is presented in Figure 4.

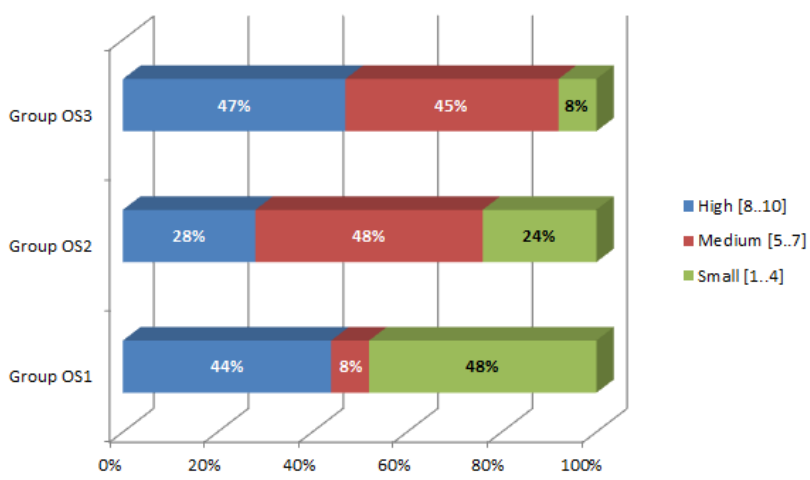

Figure 4. Graphical representation of the group's results 
As the next step, differences between the groups were analysed, regarding the grades' distribution.

Further, these obtained values were analysed using the Chi-square test. The expected results are presented in Table 2 and Figure 5.

Table 2. Expected results

\begin{tabular}{llll}
\hline \multicolumn{1}{c}{ Grades } & OS1-OS2 & OS2-OS3 & OS3-OS1 \\
\hline High [8-10] & $\chi^{2}=3.81$ & $\chi^{2}=2.38$ & $\chi^{2}=3.81$ \\
Medium [5-7] & $\chi^{2}=6.10$ & $\chi^{2}=3.81$ & $\chi^{2}=6.10$ \\
Small [1-4] & $\chi^{2}=6.10$ & $\chi^{2}=3.81$ & $\chi^{2}=6.10$ \\
\hline
\end{tabular}

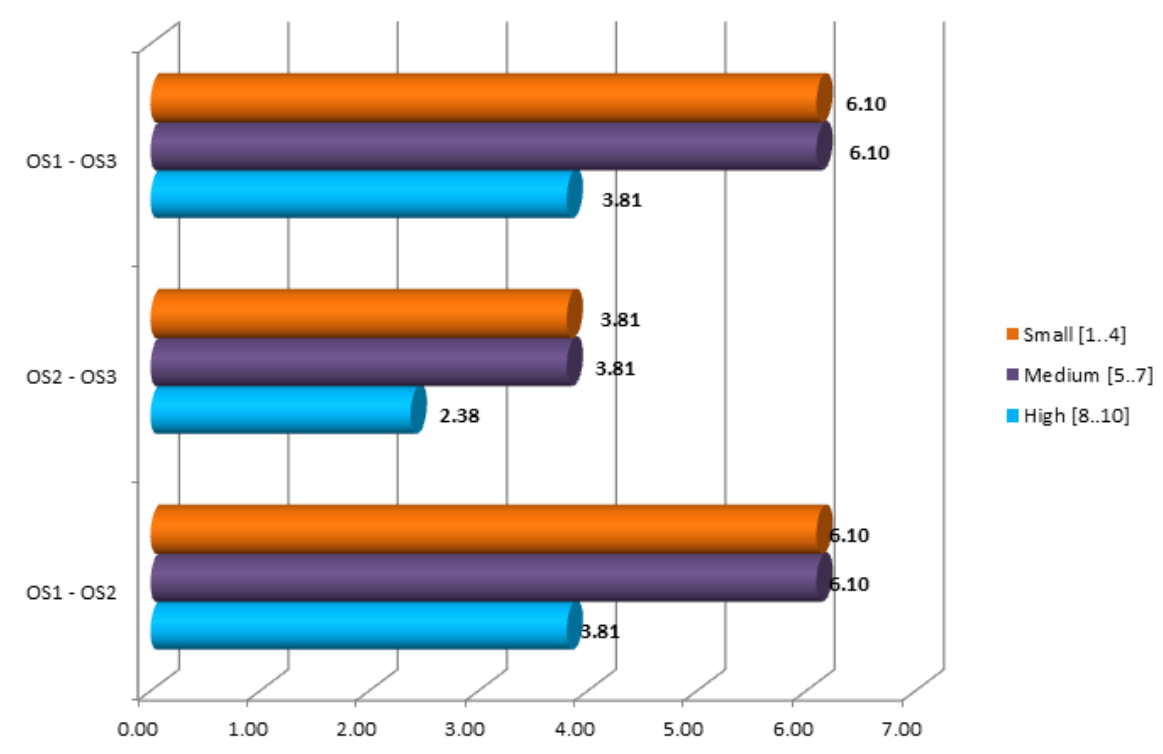

Figure 5. Chi-square test results

The $p$ value for the calculated $\chi 2$ is 0.1756 , so our hypothesis is confirmed. The deviation is small enough that chance alone accounts for it. This value means that there is a $17 \%$ probability that any deviation from the expected results is due to chance only. Of course, this is within the range of acceptable deviation (Edu, 2016).

Noticeable differences can be observed between the groups OS1 and OS3. Participants from group OS3 obtained fewer low grades and more medium grades compared to the group OS1; however, there is no difference in the highest grades. Differences between the groups OS1 and OS2 and groups OS2 and OS3 were statistically insignificant.

\section{Conclusions}

The results of the case study lead us to conclude that a combination of traditional and modern methods is more successful for operative staff training. For example, participants in group OS3 were trained online by modern methods but were offered daily support by the presence of a trainer through our online platform.

We believe that using traditional methods combined with modern ones, participants can achieve a better performance. 
Pascu, L., Simo, A. \& Vernica, A. M. (2019). Integrating Microsoft loT, machine learning in a large-scale power meter reading. International Journal of New Trends in Social Sciences. 3(1), 010-016. https://doi.org/10.18844/ijntss.v3i1.3815

\section{References}

Coskun, Z., Adiguzel, T. \& Catak, G. (2019). Acoustic labyrinth: validation of a game - based heart auscultation educational tool. World Journal on Educational Technology: Current Issues, 11(4), 245-256. Retrieved from https://doi.org/10.18844/wjet.v11i4.4394

Edu. (2016). Retrieved from http://www2.Iv.psu.edu/jxm57/irp/chisquar.html

Historical Temperature Measurements. (2016). Retrieved from http://www.wunderground.com/

Jigoria-Oprea, D., Simo, A. \& Borlea, I. (2014). AITRANS - web application for training and testing operative personnel in high voltage power substations. Proceedings 6th World Conference on Educational Sciences, University of Malta, Malta.

Metering. (2015). Retrieved from http://www.metering.com/minsen-your-ideal-supplier-of-wireless-water-gaselectricity-meters/

Next Day Prediction. (2016). Retrieved from http://www.weather.com/

Rosenberg, M. (2011). E-learning: strategies for delivering knowledge in the digital age. New York, NY: McGraw Hill.

Simo, A., Barbulescu, C. \& Kilyeni, S. (2014). Current practices in E-learning: a case study for electrical power engineering in higher education. 6th World Conference on Educational Sciences, February 6-9, University of Malta, Malta. 